$\stackrel{N / E}{=}$

Global Journals Inc

$20^{2}$

(2)

今

$2+$

GLOBAL JOURNAL OF RESEARCHES IN ENGINEERING: A

MECHANICAL AND MECHANICS ENGINEERING

Volume 21 Issue 1 Version 1.0 Year 2021

Type: Double Blind Peer Reviewed International Research Journal

Publisher: Global Journals

Online ISSN: 2249-4596 \& Print ISSN: 0975-5861

\title{
Improvement on Planar Mechanism Composition Principle
}

\section{By Ji-Chao Li \& Ji-Oiang Li}

Hoseo University

Abstract- By investigation of movement of the Assur groups in normal connecting condition, and by inspection of the kinematic pair concept, the conclusions were found that "The freedom of Assur group is zero" in the Planar Mechanism Composition Principle conflicts with the fact that Assur group can move, and the external kinematic pairs of Assur group are inconsistent with the kinematic pair concept. Proposals were put forward then that the motion characteristics of Assur group should be studied in normal connecting conditions, Grade I Linkage Group should be introduced, and the PPP Type Linkage Group existence as an example was provided. Some new views were put forward in discussion of Planar Mechanism Composition Principle. And then an example of mechanism analysis was given to show that the correct statement of the Mechanism Composition Principle is helpful to solve mechanism analysis problems.

Keywords: planar mechanism composition principle; assur group; grade I linkage group; ppp type grade II linkage group; internal connecting pair; external connecting pair.

GJRE-A Classification: FOR Code: 091399

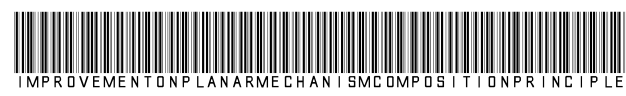

Strictly as per the compliance and regulations of:

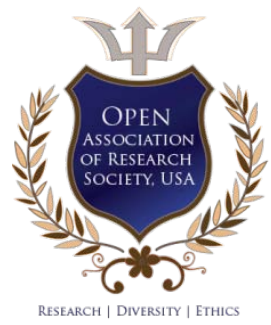

(C) 2021. Ji-Chao Li \& Ji-Qiang Li. This is a research/review paper, distributed under the terms of the Creative Commons Attribution-Noncommercial 3.0 Unported License http://creativecommons.org/licenses/by-nc/3.0/), permitting all non commercial use, distribution, and reproduction in any medium, provided the original work is properly cited. 


\title{
Improvement on Planar Mechanism Composition Principle
}

\author{
Ji-Chao $\mathrm{Li}^{\alpha} \&$ Ji-Qiang $\mathrm{Li}^{\sigma}$
}

Abstract-By investigation of movement of the Assur groups in normal connecting condition, and by inspection of the kinematic pair concept, the conclusions were found that "The freedom of Assur group is zero" in the Planar Mechanism Composition Principle conflicts with the fact that Assur group can move, and the external kinematic pairs of Assur group are inconsistent with the kinematic pair concept. Proposals were put forward then that the motion characteristics of Assur group should be studied in normal connecting conditions, Grade I Linkage Group should be introduced, and the PPP Type Linkage Group existence as an example was provided. Some new views were put forward in discussion of Planar Mechanism Composition Principle. And then an example of mechanism analysis was given to show that the correct statement of the Mechanism Composition Principle is helpful to solve mechanism analysis problems.

Keywords: planar mechanism composition principle; assur group; grade I linkage group; ppp type grade II linkage group; internal connecting pair; external connecting pair.

\section{InTRODUCTION}

1 t commonly exists such formulations in teaching materials of Mechanical Principle in universities as the member group who is the most simple, can't be disassembled further, and whose freedom is zero, is called the basic linkage group or Assur's Group [1]. Any plane lower pair mechanism can be seen as the system made up of several basic linkage groups successively connecting to the original motive parts and the frame. This is the composing principle of planar lower pair mechanism [2].

According to the concept that freedom is the number of independent motion parameters, so the system whose freedom is zero can't move. The freedom of the basic linkage group is zero [3]. But the basic linkage group is movable. How to correctly understand such self-contradictory statement? To find the origin of the problem, we should study those most basic element conception. The elements composed of the mechanism are simply members and kinematic pairs. So, it is necessary for us to make clear and unify those element conceptions to avoid ambiguity [4].

Here list only RRR, RRP, RPR, PRP, and RPP five kinds of basic linkage group in Mechanical Principle

Author a $\sigma$ : Department of Mechanical Engineering, Graduate school, Hoseo University, Asan 31499, South Korea.

e-mails: jichao@naver.com, ljq7436@naver.com textbooks, it denied the exist of PPP type basic linkage group. Whether the PPP type basic linkage group exist or not, we should not arbitrarily give a conclusion. We should judge it according to the definition and the practical examples of the basic linkage group. If the special mechanism using the PPP type basic linkage group can be found, and then the existing characteristics of the PPP type basic linkage group can be proved, and so the errors that exclude the PPP type basic linkage group can be corrected in the university textbooks $[3,5]$.

\section{The Statement of "The Components Group Whose Freedom is Zero"}

a) The conception of "Freedom"

Before two component members (member, thereafter) compose kinematic pair, they have six relative freedoms in space. After two members compose kinematic pair, the relative movement between them will be constrained. The relationship between kinematic pair freedom (expressed as "f") and constrain number (expressed as "s") is $f=6-s$ [6]. In planar motion, member 1 which has not formed kinematic pair with member 2, has three independent motions: moving along $x$ axis, moving along $y$ axis and rotating on the axis vertical to the moving plane, namely it has three freedoms. And when the two members connect with kinematic pair, the relative motions between the two members are limited. This kind of restriction is called constraint. That is the fact that kinematic pair introduces constraint and reduce the member's freedom [7].

So, the number of the independent motion parameters is the number of the freedom. If the freedom is zero, namely there is no independent motion parameter, there is no relative motions. The freedom of the basic linkage group is zero, can it move? And how to study the motion performance of the basic linkage group? As we all know that the mechanism is movable, the basic linkage group as part of it is also movable. So the difficult to judge the motion condition of zero freedom emerges.

\section{b) The conception of "Kinematic Pair"}

When members compose mechanism, various members should be connected with one another by some means, and every member should at least be connected with other one member. Obviously such connection should guarantee that there are still some 
relative motions between two connected members. Such movable connection composed by two members' direct contact is called kinematic pair $[1,6]$.

We can see that there is no kinematic pair with only one member existing, and there is also no kinematic pair when one member does not connect with other member. Separate the mechanism's frame and original motive parts connecting with the frame from follower system, and make them independent [6], this can make the kinematic pair change, for the connection has been divided, and the divided connection is not kinematic pair any more. While the basic linkage group reserves such "divided connection", I will ask if such connection is still "kinematic pair"?

\section{c) The "Kinematic Pair" in the basic linkage group}

The "kinematic pairs" in the basic linkage group have been classified. In every basic linkage group, the kinematic pairs connecting the internal members in the linkage group are called internal pairs, the kinematic pairs connecting the basic linkage group and other members are called external pairs [7], there are also conceptions as "external kinematic pairs" [9], "external connecting pairs"[10], "external end pairs" [11], "internal kinematic pairs" [6, 8], "inner pairs", "outer pairs" [12, 13].

In mechanism moving graphics, "the internal pairs" and "the external pairs" are not distinguished by different symbols. Can they be identified clearly? And can they convert into each other? As "the kinematic pairs"are movable connections composed by two members' direct contact, is there contradiction between the concepts of "external pairs" and "kinematic pairs"?

\section{ili. The Statement of "The Composing Principle of Plane Mechanism"}

a) The problem of whether the property of mechanism movement changing

Any plane lower pair mechanism can be seen as systems composed by many basic linkage groups

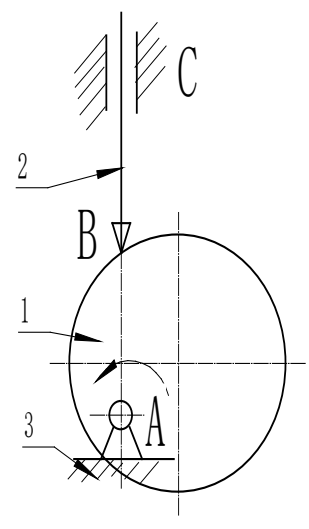

a

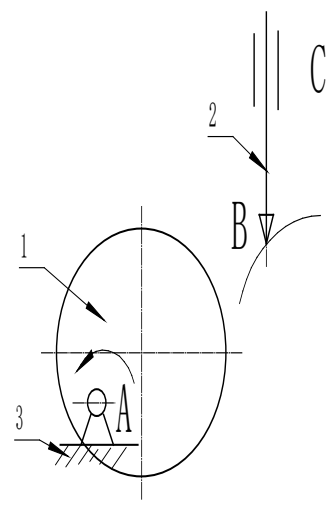

b

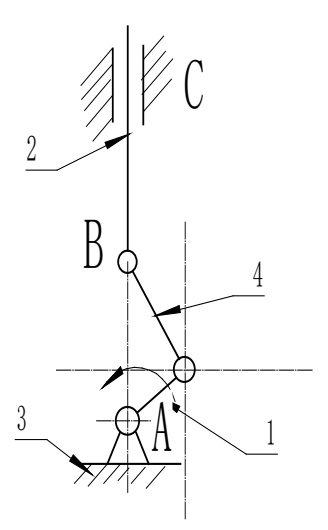

C

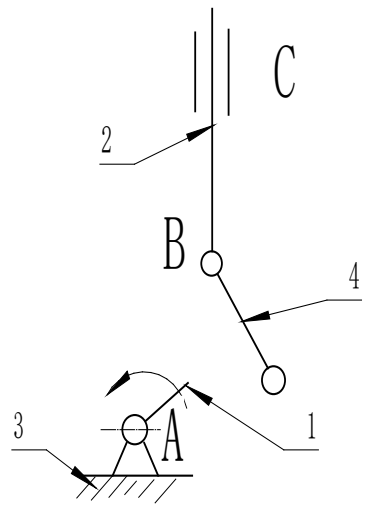

d

Figure 1: The mechanism structure: (a) a high pair mechanism; (b) anslyzing 1; (c) anslyzing 2; (d) anslyzing 3 


\section{How to Study the Performance of "The Basic Linkage Group"}

a) The premise to study the performance of basic linkage group

According to the definition of kinematic pair, strictly speaking, the external connecting pairs of the basic linkage group are only artificially assumed symbols representing kinematic pairs, which have already lost the real nature of the connection. So, to see the property of the basic linkage group, you should connect the external connecting pairs consequently to the original motive part and frame, and then those external connecting pairs have actual connecting sense, and they compose the genuine kinematic pairs.

The composing characteristics of the basic linkage group is zero freedom. It is the inevitable result people can obtain after artificially defining the basic mechanism and removing it from the whole mechanism. The basic mechanism is the members' system composed by one low pair member and the frame, and it has the same freedom as the number of the original motive parts, and it can move as well. To see whether the basic linkage group can move relatively, we should connect the external connecting pairs to the original motive part and the frame, then we can see the real properties of relative movements of the basic linkage group. Namely, we can judge the basic linkage group's relative movement properties in real connecting conditions.

\section{b) The existence of PPP type linkage group and its proof}

As a mechanism is a kinematic chain who has definitive relative movements. When any basic linkage group is connected consequently to the original mechanism and the frame, the basic linkage group should have relative motility. The basic linkage group composed of two members and three lower pairs is called grade II linkage group, and it has five types [14]. Although the freedom (F) of PPP type member group (namely the member group whose three lower pairs are all prismatic pair types, shown as figure 2) is $\mathrm{F}=3 \mathrm{n}-2 P_{L}=3 \times 2-2 \times 3=0$, but we usually do not think it as grade II linkage group. Various teaching materials list out five types of grade II linkage group, including RRR type, RRP type, RPR type, PRP type and RPP type [6-10], it excludes PPP type linkage group. The view that PPP type linkage group is not a basic linkage group composed of two members and three lower pairs is not right.

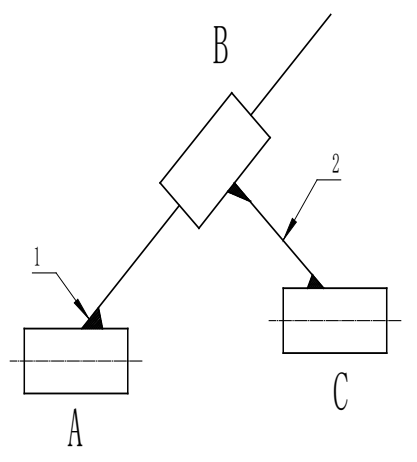

Figure 2: PPP type linkage group

To see whether the PPP type linkage group exist or not, is to see if there is such mechanism which can be composed by connecting the basic mechanism and the PPP type linkage group consequently to the original mechanism and the frame. The mechanism shown as figure 3(a) can be found, and all its kinematic pairs are movable, and it can realize determined relative motion. Its freedom $\mathrm{F}=3 \mathrm{n}-2 P_{L}-P_{H}=3 \times 5-2 \times$ $7-0=1$, the number of original motive parts. So the mechanism has definitive relative movements. The mechanism can be decomposed into structural composition shown as figure 3(b). Where the PPP type linkage group is a basic linkage group composed of two members and three lower pairs. So, the PPP type linkage group is also a basic type of grade II linkage group. 


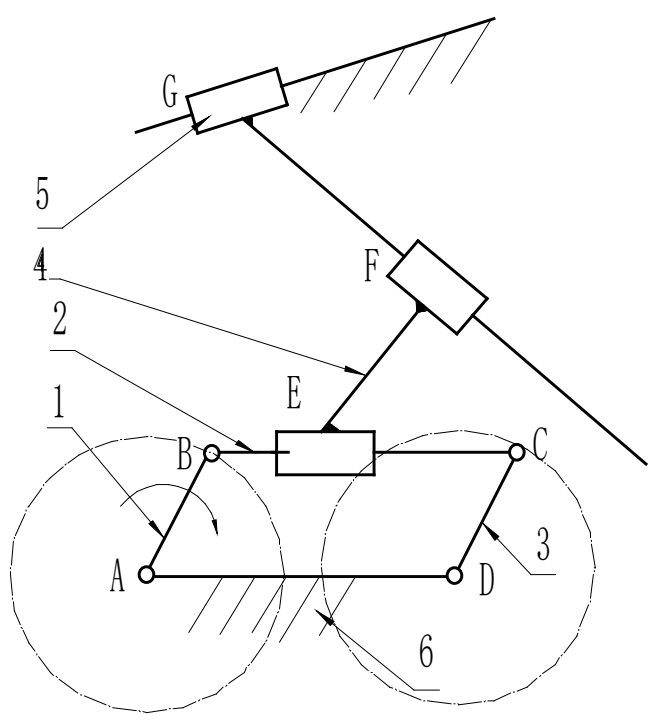

a

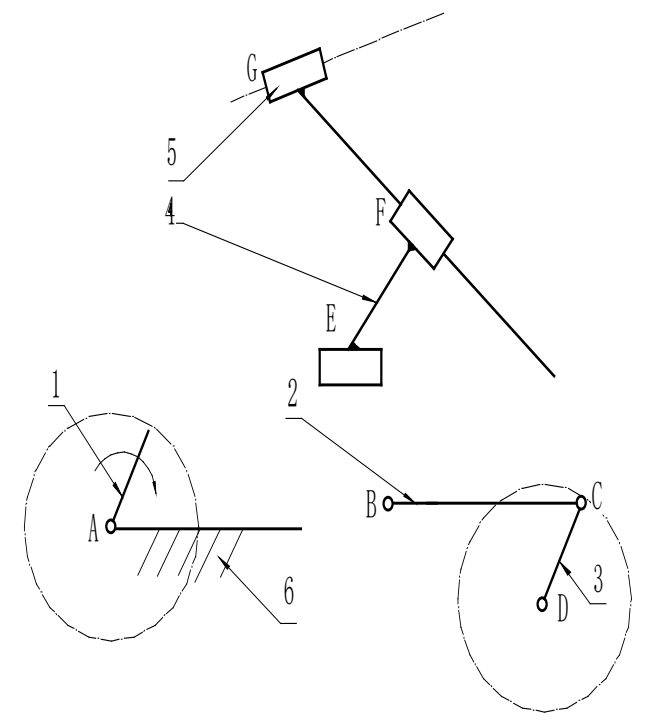

b

Figure 3: (a) an example using PPP type linkage group; (b) mechanism structural disassembling

The basic linkage group can move, and it illustrates that: the freedom of the basic linkage group exists. But the calculating result according to combination of members and constraints condition is zero. So, where has the freedom of the basic linkage group lost? Connect the basic linkage group only to one member, the connection will turn into a rigid body. Connect the basic linkage group to the basic mechanism, namely connect the external connecting pair consequently to the original motive part and the frame, while the original motive part moves according to independent motion law, it has freedom, And the freedom of the frame is zero. So, the motion properties of the basic linkage group actually depends on such connections, and the freedom of the basic linkage group depends on the original motive parts.

\section{Correction and Improvement of the \\ Planar Mechanism Composition PRINCIPLE}

In view of the above defects and deficiencies existing in the planar mechanism composition principle, correction and improvement are made on them.

a) Definition and expression of the basic linkage group

The basic linkage group is the composition of members and kinematic pairs whose freedom is zero under normal connecting conditions. The composition meets with mechanism composition formula:

$$
\mathrm{F}=3 \mathrm{n} \times 2 P_{L}-P_{H}=0
$$

Where, $\mathrm{n}$ is the number of movable members; $\mathrm{PL}$ is the number of lower pairs; $\mathrm{PH}$ is the number of higher pairs

The basic linkage group can separate subsequently from the frame and the original motive parts, but we should distinguish external connecting pair from internal connecting pair when the basic linkage group is drawn individually. The internal connecting pair is the actual existing connection between members among the basic linkage group, and it does not involve in the connection to the frame or the connection to the original motive parts, it only keep the connection among the basic linkage group itself. The external connecting pair maintains corresponding kinematic pair constraint properties, but it is not the actual existing connection when the basic linkage group is drawn individually. The external connecting pairs can only respectively involve in the connection to the frame or the connection to the original motive parts, and they can't simultaneously connect to the same one member. To distinguish the external connecting pair from the internal connecting pair, suggest the former to adopt dotted line symbols to express.

\section{b) Improvement of the planar mechanism composition principle}

i. Guarantee relative movement property unchanged

For structural analysis of planar mechanism, the concrete relative kinematic dimensions' influence on members' relative motions should be considered, to draw dotted line symbols of corresponding kinematic pair on the separating position or to preserve the component length which is corresponding to the position of the external pair, so to accurately mark down the relative positions of the kinematic pairs.

ii. The planar mechanism composition analysis process reciprocal to structural analysis process

When mechanism structural analysis (group dividing) is carried out, the process should be reciprocal to mechanism composition process. Because when composing the mechanism, the external connecting pair connects with the original motive parts or the frame, so 
when the mechanism is group-divided, such connection pair should not be cast away. In other words, no singlepair-member could emerge. And only the external connecting pair can connect with the frame or the original motive parts, the dividing group should not only meet with the mechanism composing equation (1), but also keep its internal or external connecting pair property. And because the basic linkage group should consequently connect to the original motive parts and the frame, so dividing the linkage group in the reciprocal process should not just separate the internal connecting pair or just separate the external connecting pair.

iii. Grading method of the basic linkage group

The basic linkage group's grading should according to its normal connecting condition. The higher pair element should be preserved actually within the basic linkage group, so the grade I group [15]—one member, one higher pair and one lower pair should be added. The two-linkage three-pair member group is grade II group, it has one internal connecting pair and two external connecting pairs. The four-linkage six-pair member group, which having close profile composed by three internal connecting pairs is called grade III group, which having close profile composed by four internal connecting pairs is called grade IV group [8], grade III group has a three-internal-connecting-pair three-pair linkage; grade IV group has four-internal-connectingpair characteristics, and should be recognized in the connecting conditions to the frame and the original motive parts, such property should not be changed. For the further higher Grade linkage group, because its scare application, we do not study here.

\section{iv. Non-repetition Principle}

Some component-members form kinematic pairs with the same component-member, for example, many members form kinematic pairs with the frame, we should disassemble the mechanism according to every member, every kinematic pair emerges only-one- time principle, and we only mark the frame symbol in the basic mechanism, only draw the external connecting symbol in the basic linkage group.

\section{Vi. The Value and Application of the INNOVATION THEORY}

The key of the innovation theory lies in such important studying results that the application example of PPP type grade II linkage group is found, and the existence of PPP type grade II linkage group is approved. The theory also clarify the ambiguous understanding about internal connecting pair and external connecting pair during mechanism disassembling. The theory finds an effective method to analyze the property of the basic linkage group, and find actual application basis. To use the dotted line symbol to represent the external connecting pair, and use solid line symbol to represent the internal connecting pair, it is a very simple method, and its meaning clear, and it removes communicating obstacles of ambiguous understanding and expressing problems for the development, application and improvement of the mechanism theory. So, the innovation theory has not only far-reaching theoretical significance but also important application value.

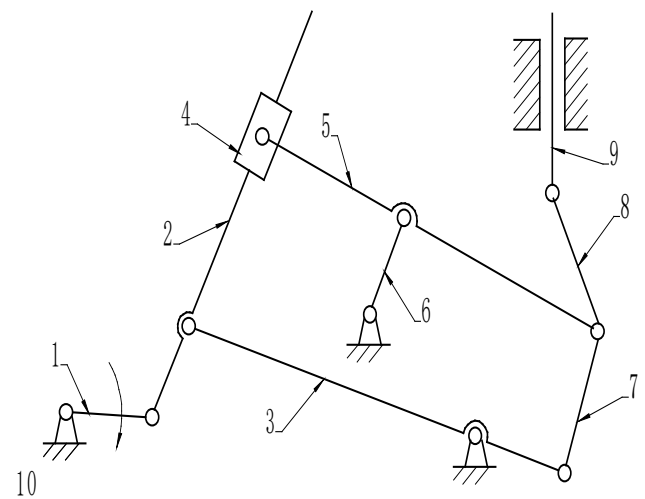

Figure 4: An example to be analyzed 


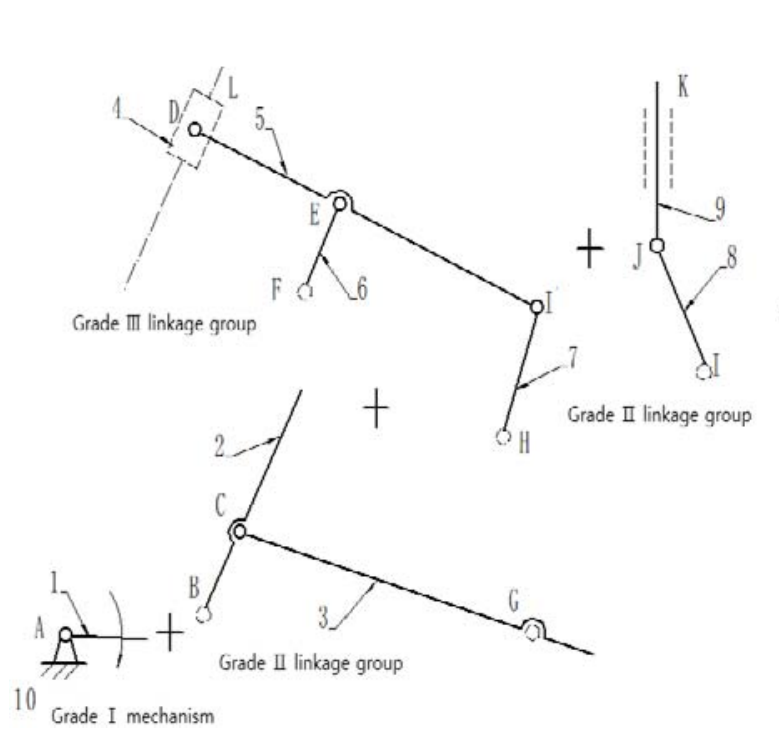

a

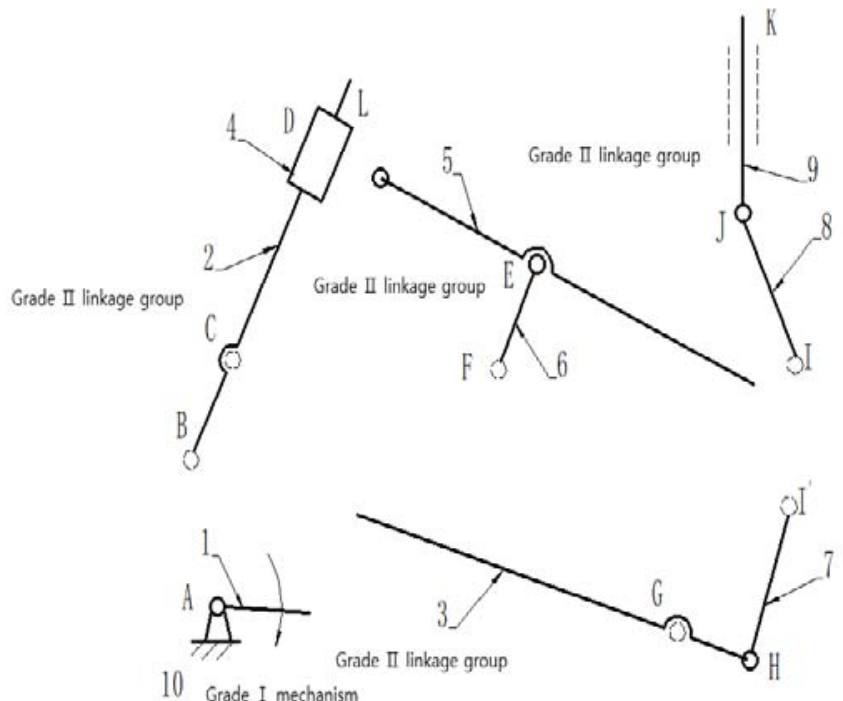

b

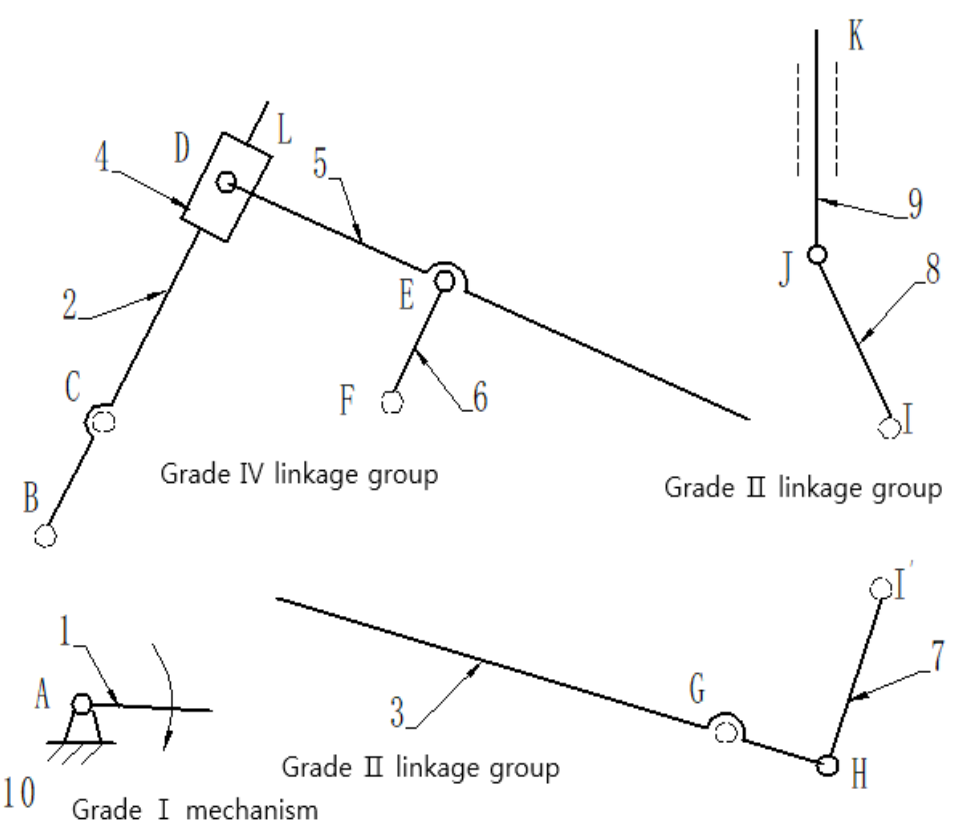

C

Figure 5: An example to be analyzed;

; (a) Result 1; (b) Results 2; (c) Results 3

Practice serves as the only standard of examining the truth. [16] For instance, we can use the above theory to analyze the structure of the mechanism shown as figure 4 [6]. We can disassemble the mechanism into the basic mechanism and many basic linkage groups as in figure 5(a). It shows that the analyzed mechanism is grade III mechanism. But if you disassemble the mechanism as figure $5(\mathrm{~b})$ and figure 5(c) to analyze the structure without considering the above theory, the results are not correct. For there are no external connecting pair which connects to the frame in 2-4 basic linkage group in figure10; there are no two external-connecting-pair structural characteristics to consistent with grade IV linkage group within 2-3-4-5-6 group in figure 5(c).
Author Contributions: Conceptualization, J.-Q.L..; methodology, J.-Q.L.; software, X.-C.L.; validation, L.C.L..; formal analysis, J.-Q.L.; investigation, X.-C.L.; resources, J.-Q.L.; data curation, J.-Q.L.; writingoriginal draft preparation, X.-C.L.; writing-review and editing, J.-Q.L.; visualization, J.-C.L.; supervision, project administration, J.-C.L ,J.-Q.L. All authors have read and agreed to the published version of the manuscript.

Funding: This research received no external funding.

\section{ACKNOWLEDGMENTS}

The authors wish to thank (1) the Korea Energy Technology Evaluation Institute (No. 20183010041940), 
(2) Hoseo University Industry-Academic Cooperation Foundation (No. 312-82-10256), (3) the Research on the Operation Mode of Practice Teaching of Mechanical Design, Manufacturing and Automation of Shandong Province, China (Project No. M2018X156), for their supports of this study.

Conflicts of Interest: The authors declare no conflict of interest

ORCID iDs

Ji-QiangLi https://orcid.org/0000-0002-2129-1252

\section{References Références Referencias}

1. Gu, J., Xie, Z., \& Wu, X. Spatial Stiffness Analysis of the Planar Parallel Part for a Hybrid Model Support Mechanism. Applied Sciences, 2020, 10(18), 6342.

2. Yamamoto, T., Iwatsuki, N., \& Ikeda, I. Automated Kinematic Analysis of Closed-Loop Planar Link Mechanisms. Machines, 2020, 8(3), 41.

3. Fomin, A., Petelin, D., \&Jahr, A. Synthesis and Analysis of a Novel Linkage Mechanism with the Helical Motion of the End-Effector. Robotics, 2020, 9(3), 53.

4. Choi, W. H., \& Takeda, Y. Displacement Analysis and Design of a (2-RRU)-URR Parallel Mechanism Performing 2R1T Output Motion for Thumb Rehabilitation. Robotics, 2020, 9(3), 67.

5. Takuma, T. Design of Tendon-Driven Mechanism Using Geometrical Condition. In Actuators (Vol. 9, No. 3, p. 48). Multidisciplinary Digital Publishing Institute.2020.

6. Sun, H., Chen, Z.M., Ge, W.J. Mechanical Principle [M].Beijing: Higher Education Press,2006.5, 6-18

7. Liu, H.Y., Zhang, M.Q., Xu, N. Mechanical Principle [M].Beijing: China Machine Press, 2013.8, 8-26

8. Guo, W.D. Mechanical Principle [M].Beijing: Science Press, 2013.1,21.

9. Wang, Z.X., Deng, Z.Q. Mechanical Principle [M]. Beijing: Higher Education Press, 2006.5,21.

10. Wang, D., Li J.Q., Li C.L., et al. Mechanical Principle Studying Guidance \&Key to Exercises [M].Beijing: Science Press,2009.8, 6.

11. Wu, Q.H., Hu, M., Chen, W.H., et al. Configuration Design \& Analysis of Radially Foldable Wheel Mechanism [J]. Machinery Design \& Manufacture, 2011, 05, 08.

12. Xu, Q. Mechanism Composition Principle and Virtual Constraint Analysis[J]. Radio \& TV University of Science and Engineering, 2000, 05, 15.

13. Ren, Y.H. Learning Guidance of "Kinematic Analysis of Planar Linkage Mechanism" [J]. Journal of Inner Mongolia Radio \& TV University, 1988, 03, 01.

14. Kong J.Y.; Sun L.B.; Liao H. Y.; et al. Synthesis and Analysis of N-links Assur Group Model based on a new Topology Method. Proceedings of the 18th
International Academic Conference on Chinese Mechanism and Machine Science[C], 2016. 07. 13.

15. Hua D. Advanced Planar Connecting Rod Mechanism- the Ninth of Mechanism Type Selection Basis[9] [J]. Machinery, 1982.09.28.

16. Lan, Z.H., Zou, H.J. High Pair Assur Group and General Planar Mechanism Composition Principle. Proceedings of the 11th National Annual Meeting of Mechanism \& the First Youth Mechanism Seminar[C]. 1998.

17. Guang,M. Daily's Special Commentator. Practice Is the Sole Criterion for Testing Truth. Guangming Daily[N] 2005, 05, 11(2/7). 